\title{
Simulation of Multi-Element Antenna Systems for Navigation Applications
}

\author{
Achim Hornbostel, Andriy Konovaltsev, Holmer Denks, and Felix Antreich
}

\begin{abstract}
The application of user terminals with multiple antenna inputs for use with the Global Navigation Satellite Systems like Global Positioning System (GPS) and Galileo has attracted more and more attention in the past years. Multiple antennas may be spread over the user platform and provide signals required for the platform attitude estimation or may be arranged in an antenna array to be used together with array processing algorithms for improving signal reception, e.g., for multi-path and interference mitigation. In order to generate signals for testing of receivers with multiple antenna inputs and corresponding receiver algorithms in a laboratory environment, a unique hardware signal simulation tool for wavefront simulation has been developed. The signals for a number of antenna elements are first generated in a flexible user defined geometry as digital signals in baseband and then mixed up to individual RF-outputs. This paper describes the principle functionality of the system and addresses some calibration issues. Measurement setups and results of data processing with simulated signals for different applications are shown and discussed.
\end{abstract}

Index Terms-Adaptive arrays, interference suppression satellite navigation systems, signal processing, simulation.

\section{INTRODUCTION}

$\mathbf{T}$ HE PERFORMANCE of positioning with Global Navigation Satellite Systems (GNSS) is sensitive to disturbances by reflected signals, e.g., from ground or buildings. Due to the low power of the received satellite signals, navigation receivers are also vulnerable for unintentional or intentional interference. For safety critical and safety of life applications, e.g., in the aviation domain, receivers must become robust against these disturbances. Spatial filtering by utilization of adaptive arrays offers a very effective way to mitigate unwanted signals and to enhance simultaneously the signal-to-noise ratio (SNR) of the desired signals. The effectiveness of spatial filtering does not strongly depend on the signal structure in contrast to most other interference mitigation techniques.

For testing of receivers with multiple antenna inputs for spatial filtering techniques, adaptive array processing, or other applications like heading and attitude determination sophisticated hardware simulation tools are required in order to generate the signals arriving at the individual antenna elements with the correct directional information. Common approaches, which have been used in the past, are the distribution of a

Manuscript received April 4, 2007; revised December 17, 2007

The authors are with the Institute of Communications and Navigation, German Aerospace Center (DLR), 82230 Wessling-Oberpfaffenhofen, Germany (e-mail: achim.hornbostel@dlr.de; andriy.konovaltsev@dlr.de; holmer.denks@dlr.de; felix.antreich@dlr.de).

Color versions of one or more of the figures in this paper are available online at http://ieeexplore.ieee.org.

Digital Object Identifier 10.1109/JSYST.2007.916048 number of RF-transmitters in an anechoic chamber or the usage of several synchronized signal generators. The purpose of this paper is to describe a new simulation approach and its application, which requires reasonably less effort and can be set up in a small laboratory. Basic assumptions are that all directional information of a signal which impinges on an antenna array is contained in the differences of its carrier phase at each antenna element, and that for narrowband signals these carrier phase shifts can be expressed as complex weighting factors applied to the baseband signals [1], [2]. Therefore, the idea of the new simulation approach is to generate the signals of individual satellites, multipath signals, and interference signals with a hardware Global Positioning System (GPS)/Galileo constellation simulator in digital baseband and to apply the different carrier phase shifts to each signal by sets of complex weighting factors. These weighting factors depend on the geometrical scenario and antenna geometry and are different for each antenna element. Finally, the weighted baseband signals for each antenna element are combined and modulated onto individual RF-carriers which are made available at individual RF-ports.

The concept of wavefront generation in baseband, as described in the previous paragraph, has been realized in a unique hardware simulation system called multiple-output advanced signal test environment for receivers (MASTER). It consists of two Spirent GSS7790 multi-output GPS/Galileo simulators, which were modified on request to make the digital I/Q baseband signals available for external processing before they are mixed up to RF, and an additional digital processing device called MATRIX manufactured by the German Aerospace Center (DLR), where the complex weighting and combination, i.e., the mapping of the generated baseband signals to the individual antenna elements, takes place. The basic theory of the wavefront generation in the digital baseband domain and the architecture of the MASTER system were already described in a previous paper [1]. For convenience, they are briefly recalled in Sections II and III of this paper, which, however, is more dedicated to the practical use of the system for specific application scenarios.

Many different methods for adaptive array processing have already been proposed and reported in the literature [3]-[14]. These include classical digital beamforming techniques as well as adaptive methods like power minimization or use of reference signals with known properties (e.g., constant modulus, pilot signals) or estimation methods like maximum-likelihood estimators or the space alternating generalized expectation-maximization (SAGE) algorithm which is presented in Section IV of this paper. For classical beamforming, the challenge for navigation is that the satellite signals or multi-path signals are below the noise floor when they arrive at the antenna. Therefore, either $a$ 
priori knowledge of the directions-of-arrival (DOA) of the signals must be available or the beamforming has to be applied after correlation. An example for DOA estimation after correlation is shown in Section IV of this paper. Another application is the attitude determination of an antenna platform with help of multiple antennas [15], [16]. An example for heading determination of a vehicle equipped with two antennas is also presented in Section IV.

This paper is organized as follows. Section II presents the data model and fundamentals of the applied theory. Section III describes the architecture, principle function, measurement setup, and calibration of the MASTER system. Section IV presents algorithms and experimental results with simulated data for different applications, in particular heading, DOA estimation, interference cancellation, and adaptive multi-path mitigation with the SAGE algorithm. Finally, some conclusions are drawn in Section V.

\section{DAtA Model}

In this section, we introduce the array signal model that is used to describe the principle operation of the wavefront generation unit of the MASTER system. This unit later on is just called MATRIX and will be described in detail in Section III. The introduced signal model is also used for the multi-antenna and array processing algorithms presented later in Section IV.

We assume that $L$ bandpass filtered narrowband signals $s_{\ell}(t) e^{-2 \pi f_{c} t}$ with planar wavefronts of carrier frequency $f_{c}$, incident azimuth $\phi_{\ell}$, and incident elevation $\vartheta_{\ell}, 1 \leq \ell \leq L$ are impinging on an array of $M, 1 \leq m \leq M$, sensor elements. The narrowband assumption implies [3] that the bandwidth of the impinging signals is limited and the amplitudes and phases of the complex envelopes of the signals $s_{\ell}(t)$ vary slowly with respect to the propagation time across the antenna array. In other words, the narrowband assumption allows the time delay of a signal across the antenna array be simply modelled as a phase shift of the carrier. Further, the transmission medium is considered linear such that the noise corrupted baseband signals at the antenna outputs $\mathbf{x}(t) \in \mathbb{C}^{M \times 1}$ can be modelled as a superposition of the $L$ impinging baseband signals $s_{\ell}(t)$ and complex temporal and spatial white Gaussian noise $\mathbf{n}(t) \in \mathbb{C}^{M \times 1}$. The impinging signals are also assumed to be generated by $L$ point sources which are located far from the antenna array such that the direction of propagation is practically equal at each array element and the wavefronts can be considered as planar (far-field approximation). Thus, the baseband representation of the antenna array output can be written as follows:

$$
\mathbf{x}(t)=\sum_{\ell=1}^{L} \mathbf{a}\left(\phi_{\ell}(t), \vartheta_{\ell}(t)\right) s_{\ell}(t)+\mathbf{n}(t) .
$$

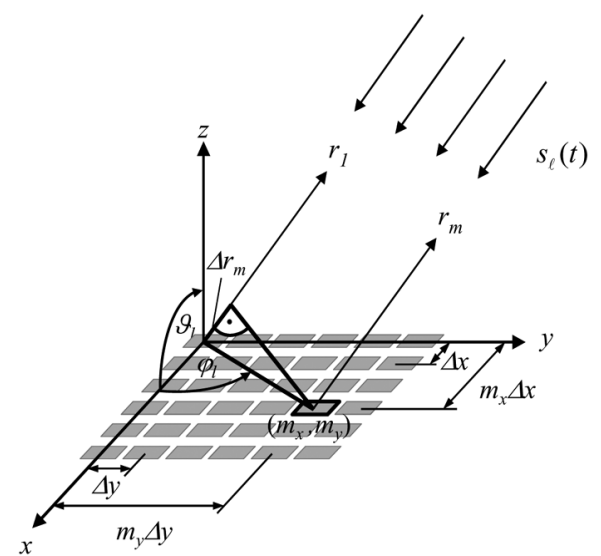

Fig. 1. Array geometry.

Here, $\mathbf{a}\left(\phi_{\ell}(t), \vartheta_{\ell}(t)\right)$ denotes the steering vector of the antenna array, $\phi_{\ell}(t)$ the azimuth, and $\vartheta_{\ell}(t)$ the elevation angle of the signal $s_{\ell}(t)$ at time $t$ in the local antenna coordinate system. In general, the steering vector or array manifold vector is defined in (2), shown at the bottom of the page,

where $F_{m}\left(\phi_{\ell}(t), \vartheta_{\ell}(t)\right)$ denotes the complex directivity pattern of the $m$ th of $M$ antenna array elements. Here, $\xi_{m}(t)$ is the carrier phase offset occurred at the $m$ th array element during the propagation of a signal wavefront along the array aperture

$$
\begin{aligned}
& \xi_{m}=\frac{2 \pi}{\lambda}\left(x_{m} \cos \phi_{\ell}(t) \cos \vartheta_{\ell}(t)\right. \\
& \left.\quad+y_{m} \sin \phi_{\ell}(t) \cos \vartheta_{\ell}(t)+z_{m} \sin \vartheta_{\ell}(t)\right)
\end{aligned}
$$

where $\lambda=\left(c / f_{c}\right)$ is the carrier wavelength and $c$ is the effective propagation speed of signals at frequency $f_{c}$ across the array aperture. Here, $x_{m}, y_{m}$, and $z_{m}$ are the Cartesian coordinates of the $m$ th sensor element in the local antenna coordinate system, as depicted in Fig. 1.

Especially, for a uniform rectangular array (URA) of $M=$ $M_{x} M_{y}$ omnidirectional sensors with $M_{x}$ elements being displaced by $\Delta x$ along the $x$-axis and $M_{y}$ elements displaced by $\Delta y$ along the $y$-axis the steering vector is

$$
\begin{aligned}
& \mathbf{a}\left(\phi_{\ell}(\mathbf{t}), \vartheta_{\ell}(\mathbf{t})\right) \\
& =\left[1, \ldots, e^{j 2 \pi\left(m_{x} u_{\ell}+m_{y} v_{\ell}\right)}, \ldots, e^{j 2 \pi\left(\left(M_{x}-1\right) u_{\ell}+\left(M_{y}-1\right) v_{\ell}\right)}\right]^{T}
\end{aligned}
$$

with $0 \leq m_{x} \leq M_{x}-1,0 \leq m_{y} \leq M_{y}-1$, where

$$
u_{\ell}=\frac{\Delta x}{\lambda} \cos \phi_{\ell}(t) \cos \vartheta_{\ell}(t), \quad v_{\ell}=\frac{\Delta y}{\lambda} \sin \phi_{\ell}(t) \cos \vartheta_{\ell}(t) .
$$

Please note that the $L$ impinging signals in (1) can represent line-of-sight signals from the navigation satellites as well as re-

$$
\mathbf{a}\left(\phi_{\ell}(t), \vartheta_{\ell}(t)\right)=\left[F_{1}\left(\phi_{\ell}(t), \vartheta_{\ell}(t)\right) e^{j \xi_{1}(t)} \quad F_{2}\left(\phi_{\ell}(t), \vartheta_{\ell}(t)\right) e^{j \xi_{2}(t)} \quad \ldots \quad F_{M}\left(\phi_{\ell}(t), \vartheta_{\ell}(t)\right) e^{j \xi_{M}(t)}\right]^{T}
$$


flective components of the satellite signals (i.e., multi-path) and RF interference.

If $s_{\ell}(t)$ represents a navigation satellite signal or a reflective component of a navigation satellite signal, then we can write

$$
s_{\ell}(t)=\gamma_{\ell} \quad c\left(t-\tau_{\ell}\right)
$$

where $c\left(t-\tau_{\ell}\right)$ is a pseudorandom-noise (PRN) sequence with delay $\tau_{\ell}$ and $\gamma_{\ell}$ is the complex amplitude. In this paper, we apply Gold codes as used for the GPS C/A civil signal with a code period of $T=1 \mathrm{~ms}$ and 1023 chips per code period, each with time duration [24].

The wavefront generation unit (MATRIX) operates on digital signals. In the discrete-time notation, the array output is described as $\mathbf{x}[n]=\mathbf{x}\left(n \cdot T_{s}\right)$ with $T_{s}$ denoting the sampling interval, and the (1) can be rewritten as (6), shown at the bottom of the page.

The multiplication of the input signal vector $\mathbf{s}[n]$ with the array manifold matrix $\mathbf{A} \in \mathbb{C}^{M \times L}$ is finally the task to be realized by MATRIX as part of the MASTER simulation system.

\section{MASTER SimUlation SYSTEM}

In this section, the architecture, measurement setup, and calibration of the MASTER are described. The last subsection provides details about the data recording for offline processing.

The MASTER simulation system is especially designed for the investigation of array processing techniques in navigation receivers. It provides complete control over the physical RF signals fed into a receiver under test. Depending on the configuration of the simulation system, the RF signals contain, in general, all satellites visible at that time in the (simulated) nominal constellation and are affected by common errors. For a satellite navigation system, these errors typically are clock errors, satellite track errors, ionospheric and tropospheric errors, and multi-path. Also, the relative movement of the receiver in respect to each satellite is simulated and the resulting Doppler shift is included in the provided signal. Additionally, MASTER can generate simple continuous wave $(\mathrm{CW})$ and noise interferers. With the digital wavefront matrix (MATRIX), which is part of the MASTER simulation system, the individual satellite, multi-path, and/or interference signals are mapped to the elements of an antenna array, which can be freely configured in the control SW.

\section{A. Measurement Setup}

The general measurement setup consists of the MASTER system including the signal generator boxes, the control PC, and the up-converters, connected to the receiver under test or a data recording unit. Optional parts of MASTER are a vector signal

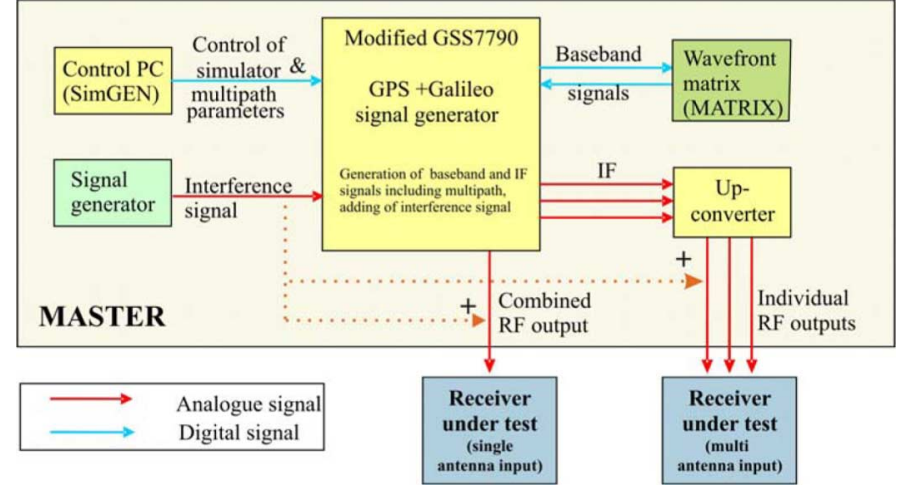

Fig. 2. Schematic overview of MASTER.

generator and the digital wavefront matrix, which we will just call MATRIX for the rest of this paper. A schematic overview of the system is given by Fig. 2 .

The core of MASTER consists of two modified GSS7790 multi-output full constellation simulators built by Spirent Communications Ltd. which provide all GPS (L1, L2, L5) and Galileo (E1, E5, E6) satellite signals as digital baseband signals. The reason to use two modified GSS7790 is two-folded. First, it simply doubles the number of channels available. Second, it enables the simulator to produce a mixed GNSS system consisting of GPS and Galileo.

One important feature of MASTER is the availability of the baseband signals via an LVDS interface which enables a manipulation of the satellite signals by MATRIX. This manipulation can be represented by a multiplication of the GNSS I/Q signals provided by the simulator with complex coefficients rated up to $100 \mathrm{~Hz}$. The main application of MATRIX is to produce phase shifts occurring due to signal propagation at individual antenna elements of an antenna array and thus generating a wavefront as described in Section II. For implementation details see [1]. Furthermore, MATRIX also enables the reallocation of individual satellite signals from the simulated constellation to specific hardware channels. For instance, in order to produce satellite signals as they are received by a $2 \times 2$ elements antenna MATRIX uses both properties as it is illustrated in Fig. 3, where 12 inputs and 4 of 12 outputs of MATRIX are used. The weighting coefficients can be updated with $100 \mathrm{~Hz}$. In case no dynamic effects have to be considered, e.g., in a scenario where the satellites and the receiver are static, a continuous update of the coefficients is not required, i.e., only one data set is sufficient.

MASTER is controlled by Spirent's SimGEN SW running on a control PC. SimGEN enables the user to define a simulation environment including parameters such as orbit parameters

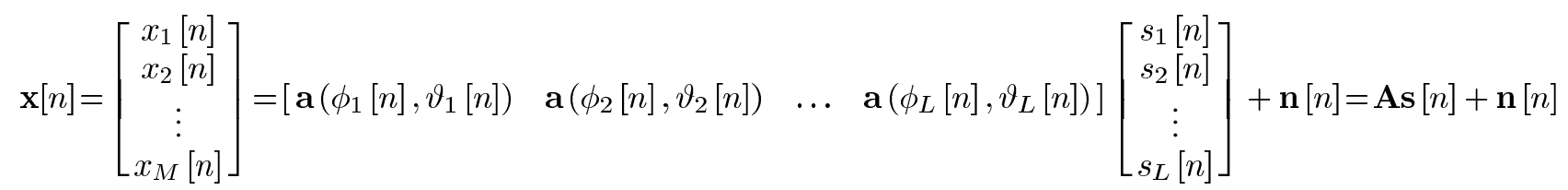




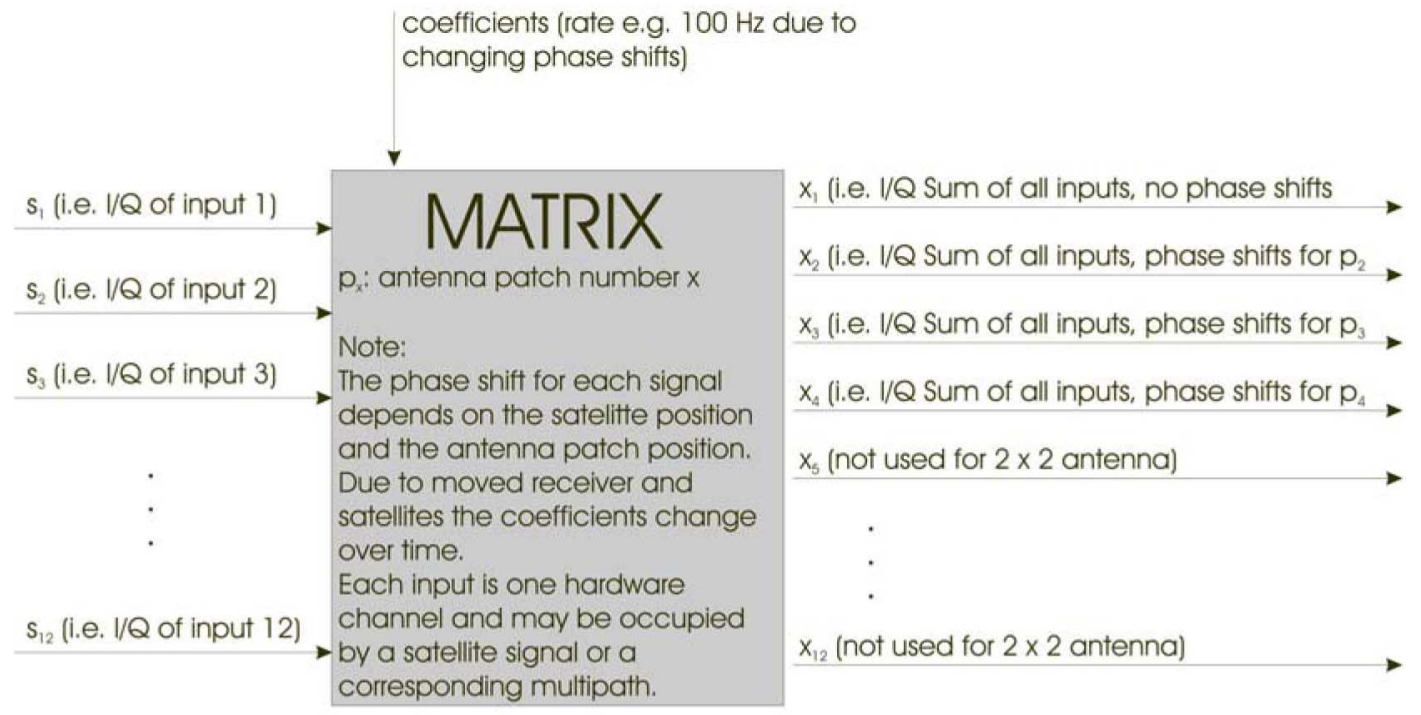

Fig. 3. Example of MATRIX operation for a $2 \times 2$ antenna $(L=12, M=4)$.

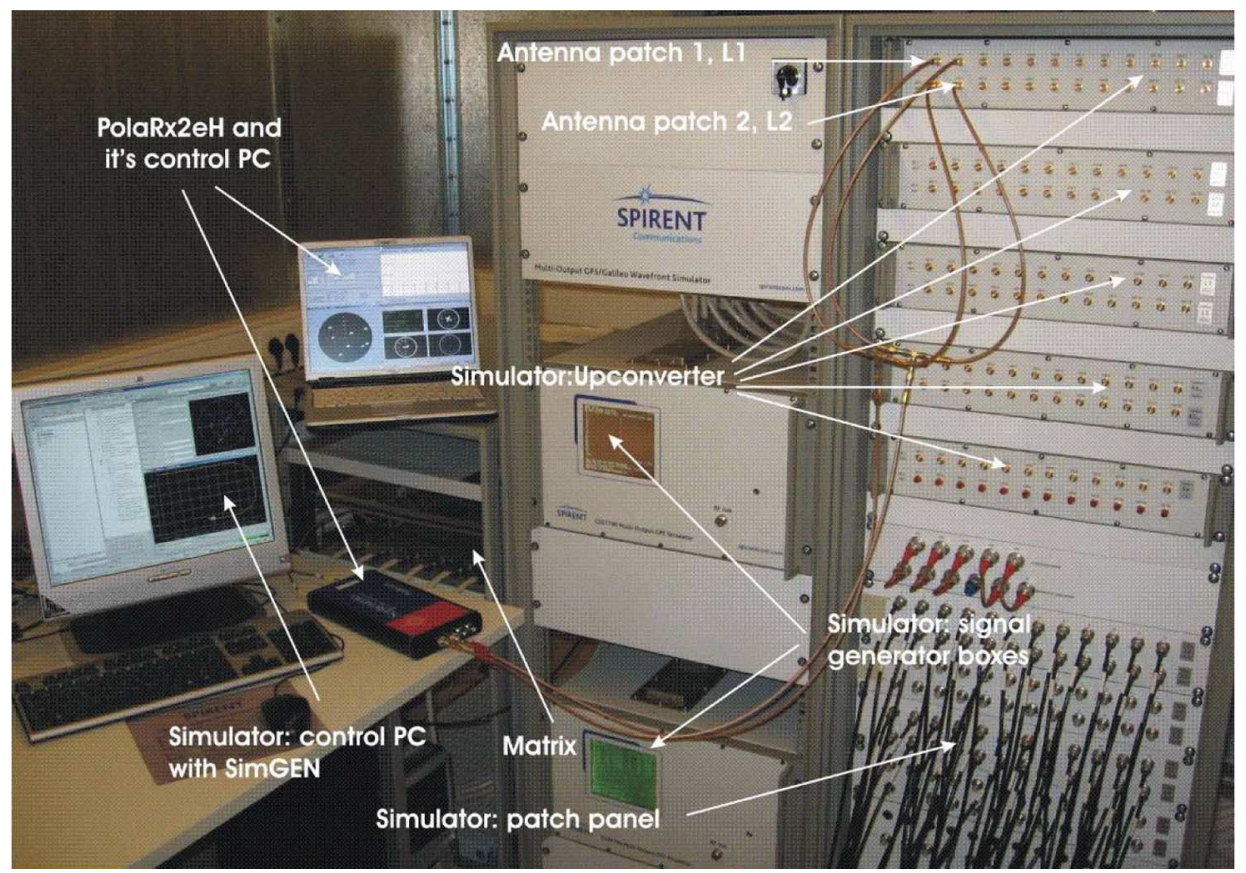

Fig. 4. Overview of the measurement setup.

of the GNSS used, clock errors, iono- and tropospheric effects, antenna pattern, multi-path, and user trajectories. It is also used to define the satellite in view (SV) signal and its components as navigation data, pilot/data channel, and modulation scheme according to the desired frequency band. The coefficients for MATRIX are generated on the same PC in a prerun of the simulation and then sent to MATRIX via an USB interface during a second simulation run.

For the desired satellite signals, two different signal paths are available: all signals provided on one combined RF output or each signal on an individual output. In the first case, the signals generated in baseband and optionally manipulated by MATRIX are combined and mixed to a common IF for all GPS and Galileo frequency bands, put on the appropriate carrier and fi- nally filtered. They are available on the combined output at a nominal power level of about $-130 \mathrm{dBm}$ and a dynamic range of $\pm 20 \mathrm{~dB}$. The combined signal can be directly fed into the RF input port of a single antenna GNSS receiver. The second case is much more powerful. The signals are each individually available on individual IF ports on the lower part of a patch panel (compare Fig. 4). Then they are mapped by BNC cables to the desired RF up-converter to be mixed up to RF. Finally, the signals are available on individual RF outputs representing either one satellite signal or, in case of multi-antenna receiver simulation with MATRIX, the superposition of all satellite signals corresponding to one antenna element. The power of the GNSS signals at these ports is approximately $40 \mathrm{~dB}$ higher than at the combined output. 
Another important feature of MASTER is to provide GNSS signals overlaid with other signals, i.e., interferers, in the same frequency range. There are two different ways of providing the user with interfering signals. The first way is to generate these signals within the modified GSS7790 simulator boxes. The user can manipulate the interferers in the same way as the satellite signals by MATRIX and tap them at the appropriate RF outputs. The drawback of this method is the limited maximal power of the signals at the RF output of up to $-60 \mathrm{dBm}$. Besides this, also the types of interferers are limited to $\mathrm{CW}$ and broadband interferers. To overcome these limitations, a powerful programmable signal generator (Agilent E8267D) is used instead. With this signal generator, signals can be generated almost arbitrarily. The drawback here is that phase shifted copies of a signal for multiple antenna outputs would have to be created at the RF level, which is not foreseen with the current measurement setup. Therefore, in the applications presented in Section IV of this paper, we did not use this feature. An alternative, which will be further worked out and tested in the future, is to generate or to record digital I/Q baseband interference signals and feed them into one input of MATRIX at the appropriate sampling rate together with the I/Q satellite baseband signals at the other inputs of MATRIX. Then the introduction of phase shifts and mapping to the individual antenna elements of the interfering signal can be performed within MATRIX in the same way as for the satellite signals.

An example of the setup for the heading experiment, which is presented in detail in Section IV-A, is shown in Fig. 4. In this case, no interferers are simulated. The device under test is a dual frequency (L1/L2) dual antenna GPS receiver (Septentrio's PolaRx2eH). The coefficients for MATRIX are calculated in such a way that the signal for the first antenna element, i.e., the sum of all visible satellite signals without any modification, is mapped to the first GPS L1, respectively, first GPS L2 RF port. On the second L1, respectively, L2 port the same signals appear but now with additional phase shifts as expected due to the position of the second simulated antenna element; the first element serves as reference. In this case, we calculated the coefficients for an antenna patch located in a distance of half a wavelength at L1 apart from the reference element. Recall, that even for the same antenna element and same satellite the phase shifts are different for L1 and L2 due to the different carrier wavelengths. Therefore, four RF outputs are required. On each of the four used RF ports all visible satellites are combined. The differences are in respect to phase and frequency. For more details, see Section IV-A.

\section{B. Calibration}

In general, MASTER provides its analogue signals at different outputs at different frequencies. Available are a combined RF output, a combined IF output, individual IF, and individual RF outputs. The SimGEN software supplies auto-calibration and interbox calibration utilities for calibration of code phases and carrier phases on individual IF and on the combined IF outputs. However, these calibration utilities do not include the phase offsets of the individual RF outputs. Therefore, these outputs have to be calibrated externally. The phase offsets depend on the chosen hardware configuration, i.e., on the signal configuration of the two simulator boxes. Although the absolute phases of the individual RF signals are drifting, the relative phases between the individual outputs are stable and reproducible when the auto-calibration was performed after power-on

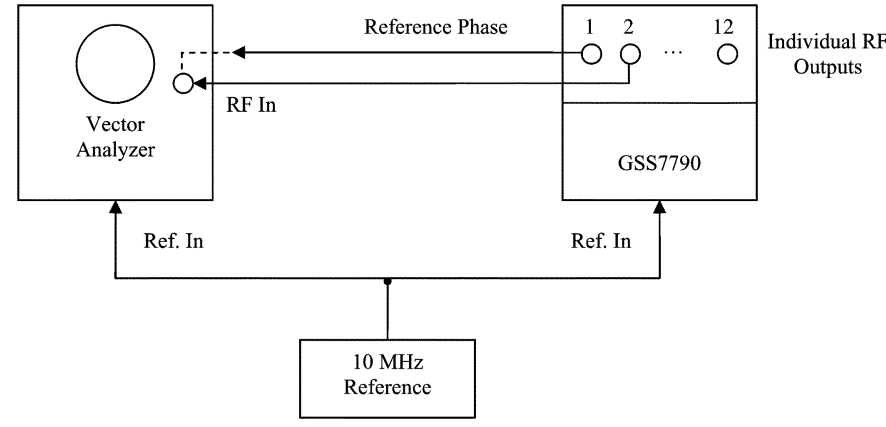

Fig. 5. Measurement setup for phase calibration.

of the system. Since for wavefront simulation and array processing only the relative phases between the antenna elements matter, the stability of the relative phases is sufficient. The relative phase offsets can be compensated by applying appropriate complex weights onto the digital baseband signals by MATRIX. Practically, the phase offsets were measured with a vector spectrum analyzer (Agilent 4443A) for each hardware configuration of interest and stored in a calibration file. This file is called by the software which computes the complex coefficients used for the wavefront generation with MATRIX.

The measurement setup for the phase calibration is shown in Fig. 5. The simulator boxes and the spectrum analyzer are both connected to an external 10-MHz reference clock. All phase offsets were measured relative to the first channel of the individual RF-boxes; i.e., first the absolute phase of the first channel was measured, then the individual relative phase offsets to the next channels were determined. It is important to measure again the absolute phase of the first channel for the calibration of each individual channel of interest due to slow but visible drifts during the time required for the measurements. The accuracy and repeatability of the relative phase measurements after some days is about $3^{\circ}$.

The approach to do the phase calibration with the help of a spectrum analyser as described above, gives very good results when the equipment to be tested has the same input impedance as the spectrum analyzer. Practically, the matching conditions often differ. This results in distortions of the signal wavefronts generated by MATRIX. The effect is similar to the phase distortions an antenna array system has to cope with in practice. However, this is not desirable when testing array processing algorithms in a controlled reference signal environment. More important, the calibration of the MASTER RF outputs cannot take into account imperfections of the receiver front end of the device under test. Therefore, a second calibration method is described in the following, which is, in particular, suitable for offline processing of recorded data in a software receiver.

In case array processing algorithms are implemented in a GNSS software receiver, the quality of the phase calibration procedure can be significantly improved by measuring the carrier phases of the individual antenna signals in the GNSS software receiver using the digital signals provided by its RF front end. One implementation of this approach when using I/Q outputs of the receiver prompt correlators is shown in Fig. 6. Since the same carrier signal reference is used in the different antenna processing channels, the difference between carrier phases in these channels for a test signal from a known direction should match the theoretical values defined by the array manifold vector. 


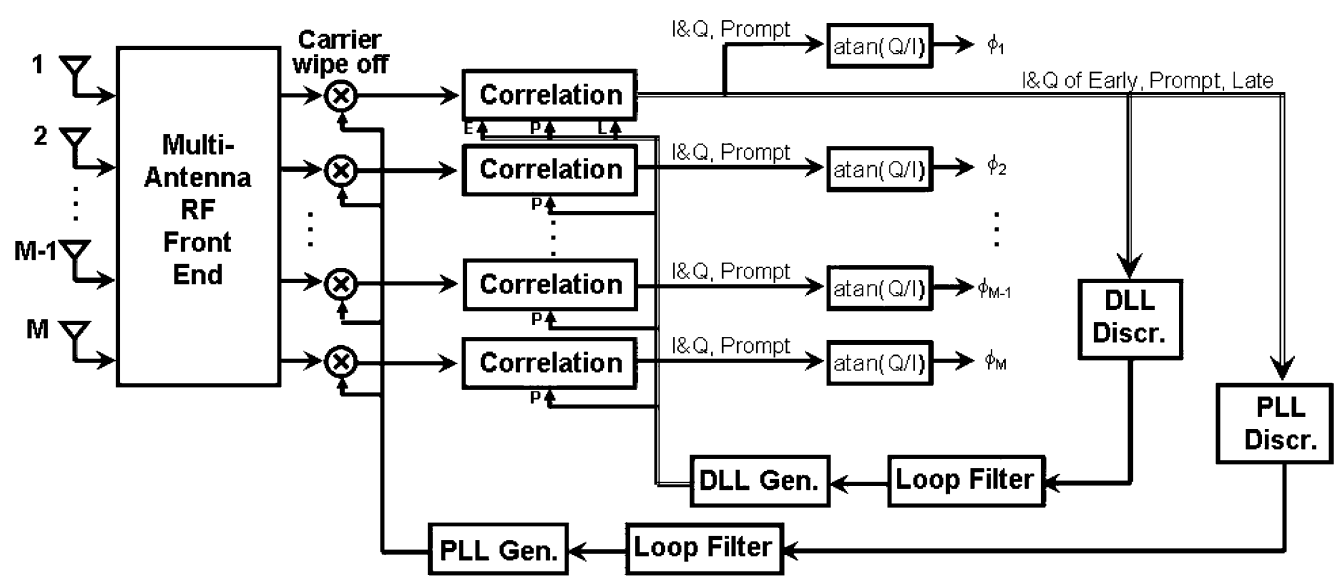

Fig. 6. Phase calibration with I/Q prompt correlator outputs in a software defined GNSS receiver.

Using this approach, one accounts for the phase distortions due to the nonperfect matching between the RF up-converter of MASTER and the receiver RF front end as well as for the phase and amplitude distortions in the RF front end itself. Another advantage of this approach is that the expensive spectrum analyzer equipment is no longer required.

\section{Data Recording}

A comparable easy way to record GNSS signals in the L1 band is to use NordNav's GPS L1 "Quad Front End." This front end is designed to operate with NordNav's software receiver but it is also possible to use it stand alone for data recording. It samples up to four independent GNSS signals with a sampling rate of $16.3676 \mathrm{MHz}$ in a $2 \mathrm{MHz}, 3 \mathrm{~dB}$ bandwidth. The data are quantized with 2 bits and down converted to an IF of 4.1304 MHz. These data are then recorded in up to four separate but synchronous files. In Fig. 7, the setup for simple L1 recording for a four antenna case is depicted. The four antenna inputs are connected to four different individual RF outputs of MASTER. The simulator and especially MATRIX are configured to output a signal corresponding to a $2 \times 2$ patch antenna with the elements spaced by half a wavelength from each other. The individual RF outputs of the simulator were calibrated in respect to phase (compare Section III-B "Calibration"). The spatial information here was completely incorporated by MATRIX. Here, the elevation and azimuth of the SV were not read from SimGEN in a prerun as in a typical test case for receivers (see, e.g., Section III-A), but artificially fixed and the DOAs were introduced by constant coefficients in MATRIX. The advantage of this method is that the position of the SV is easily determined and also fixed in respect to time which avoids in combination with a static receiver any Doppler. The disadvantage is that the location of the SV indicated in its navigation messages does not match with the phase shifts introduced by MATRIX due to its artificially fixed DOA. As long as the receiver does not exploit the navigation message, this will not cause any problems.

In a similar way, data for $\mathrm{L} 1$ and additional interferers were recorded as shown in Fig. 8. One problem which had to be solved in the simulation was to produce the quite different power levels required for the interferers in respect to the GNSS signals. In addition to the dynamic range of $40 \mathrm{~dB}$ of the power provided by the simulator hardware itself, we get here another $40 \mathrm{~dB}$ due

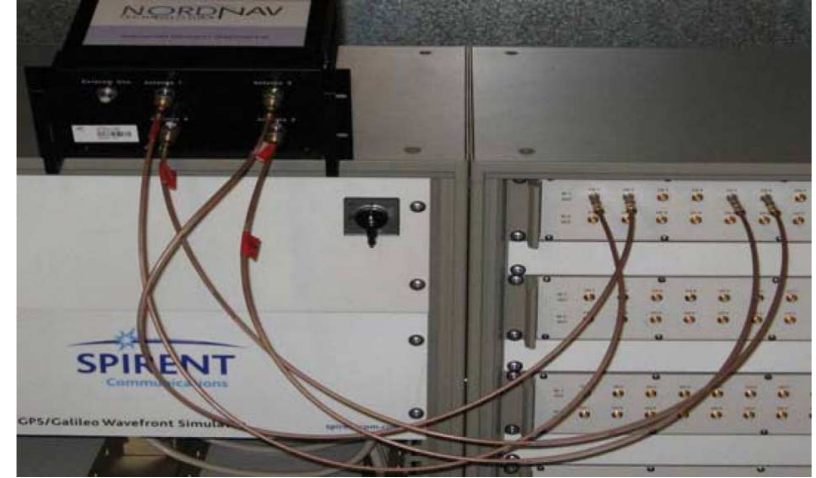

Fig. 7. NordNav's "Quad Front End” connected to MASTER for L1 recording.

to the fact that all signals available at the individual RF ports are approximately $40 \mathrm{~dB}$ above the nominal GNSS power level. In order to make use of this $40 \mathrm{~dB}$ additional power, interferers and satellite signals are routed to different baseband outputs by MATRIX and combined later at RF. The GNSS signals are reduced to the nominal GNSS-level by insertion of 40-dB attenuators, whereas the interferers are left on the high power level.

\section{APPLICATIONS}

In this section, results of different experiments are presented, which have been carried out with MASTER for different applications. These are in the order of Sections IV-A-IV-D. Determination of receiver heading, DOA estimation, interference cancellation, and maximum likelihood estimation of signal parameters with the SAGE algorithm.

\section{A. Heading}

By measuring the carrier phase differences between two antennas, the heading of a receiver or of a vehicle can be determined from the known antenna positions. A receiver with such capability is the Septentrio PolaRX2eH receiver with two antenna inputs. It determines the heading either by the antenna carrier phase differences of single frequency (GPS L1) or double frequency measurements (GPS L1 and L2). The details about the receiver algorithms can be found in [15] and [16]. 


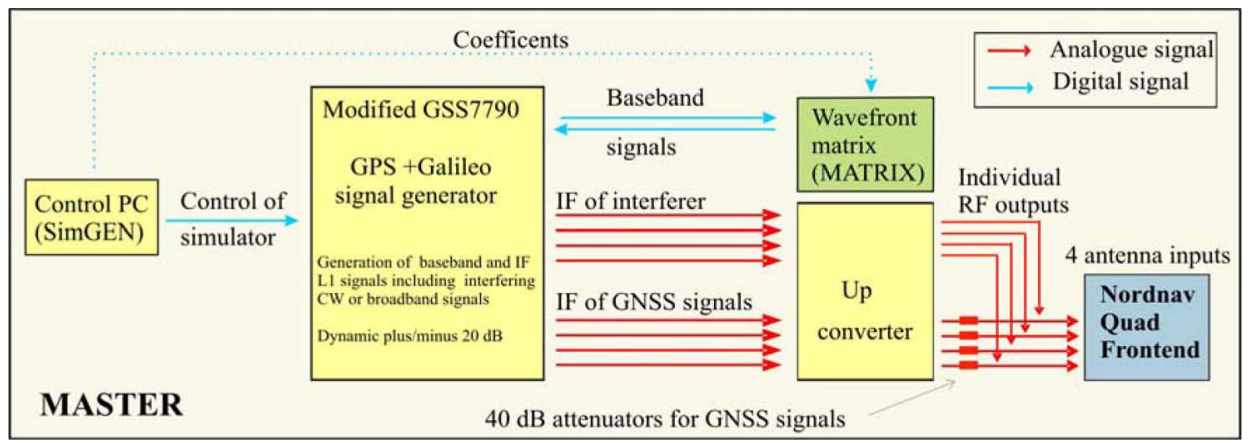

Fig. 8. Data recording with interference with "Quad Front End."
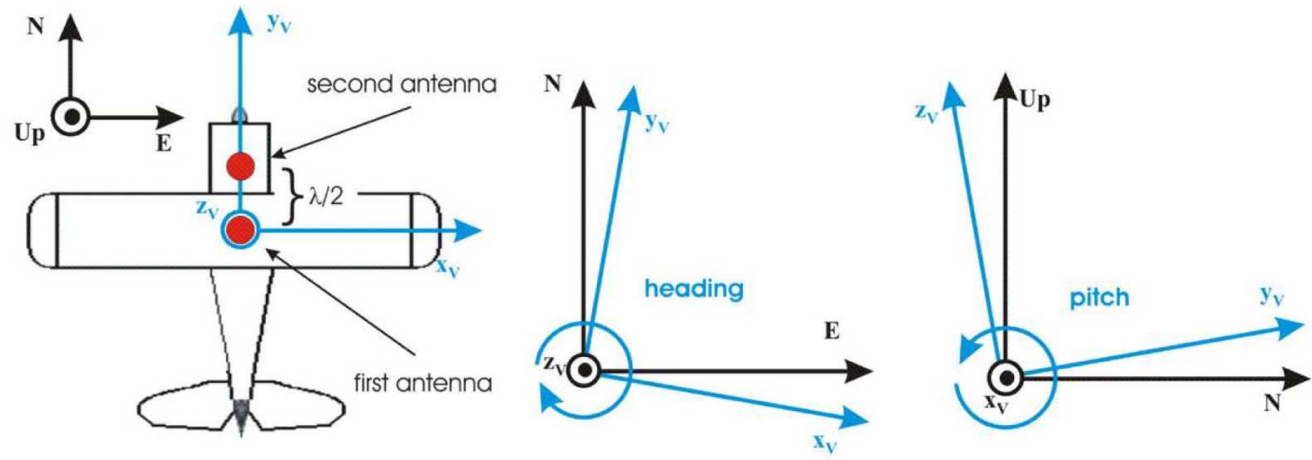

Fig. 9. Array antenna on vehicle. Local coordinate system is blue, the fixed one is black [17].

For demonstration, we created a simulation scenario with a vehicle which follows a track with several turns. The simulated $1 \times 2$ array antenna was placed in heading direction of the vehicle with a spacing of half a carrier wavelength in respect to GPS L1. Besides the geometric arrangement of the antenna elements in Fig. 9 two coordinate systems are introduced: A local one which moves with the vehicle and a fixed one. If the vehicle is heading to north the $y$-axis of the right-handed local system is directing to north.

Considering the assumption that the antenna array does not change its roll or pitch angle, the phase differences between the two antenna patches depend on the azimuth and elevation of the received satellite signals $s_{q}\left(\phi_{q}, \vartheta_{q}\right)$ and are determined by [1]

$$
\begin{aligned}
\xi_{2}(q) & =\xi_{2}\left(\phi_{q}, \vartheta_{q}\right)=2 \pi f \tau\left(\phi_{q}, \vartheta_{q}\right)=\frac{2 \pi}{\lambda} \Delta r\left(\phi_{q}, \vartheta_{q}\right) \\
& =\frac{2 \pi}{\lambda}\left(\Delta_{x} \cos \phi_{q} \cos \vartheta_{q}+\Delta_{y} \sin \phi_{q} \cos \vartheta_{q}\right)
\end{aligned}
$$

where $\Delta r$ is the geometrical difference of the signal paths to antennas 1 and 2, $\tau$ the corresponding time delay, and $\phi_{q}$ and $\vartheta_{q}$ are the azimuth and elevation angle of the received signal in the local moving coordinate system of the antennas, compare (3) with $z_{m}=0$. Using the given geometry (7) reduces to

$$
\xi_{2}(q)=\frac{2 \pi}{\lambda} \Delta y \sin \phi_{q} \cos \vartheta_{q} .
$$

Since we define the vehicle always aligned to the local horizon (i.e., pitch and roll are zero), the elevation angle $\vartheta_{q}$ of a satellite $q$ in the local antenna coordinate system, i.e., the blue one in Fig. 9, is equal to the elevation angle $\vartheta_{\text {fixed } q}$ of the satellite signals in the fixed coordinate system.

The azimuth angle $\phi_{q}$ is the difference of the satellite azimuth $\phi_{\text {fixed }, q}$ in the fixed local coordinate system and the actual heading direction of the vehicle $\phi_{\text {head }}$

$$
\phi_{q}=\phi_{\text {fixed }, q}-\phi_{\text {head }} .
$$

All three directions (satellite azimuth, satellite elevation, and vehicle heading) were read from the simulator control software SimGEN during a prerun of the simulation with a rate of $10 \mathrm{~Hz}$. The complex weighting coefficients $\cos \left(\xi_{2}(q)\right)-j \sin \left(\xi_{2}(q)\right)$ [1] for the second antenna patch were calculated for frequency bands $\mathrm{L} 1$ and $\mathrm{L} 2$ with $\xi_{2}(q)$ according to the previous formulas. Note, that the complex weighting coefficients for the first antenna patch are always $(1+0 j)$, because it is used as reference in the origin of the local antenna coordinate system. The weighting coefficients for both antenna elements were stored in a file and applied in the (second) simulation by MATRIX to the baseband I and Q satellite signals provided by the simulator. Then the signals of the different satellites were combined for one antenna element for each frequency separately and mixed up to RF in order to make them available at four individual outputs (2 L1 and $2 \mathrm{~L} 2$ ) representing the two antenna elements at the two carrier frequencies as described in Section III-A. Finally, the two RF-outputs at L1 and L2 belonging to the same antenna were combined on RF level and fed into the corresponding antenna inputs of the PolaRx2He receiver. For better matching to the $50-\Omega$ wave impedance, on each port T-combiner attenuators were inserted and this additional attenuation compensated by 


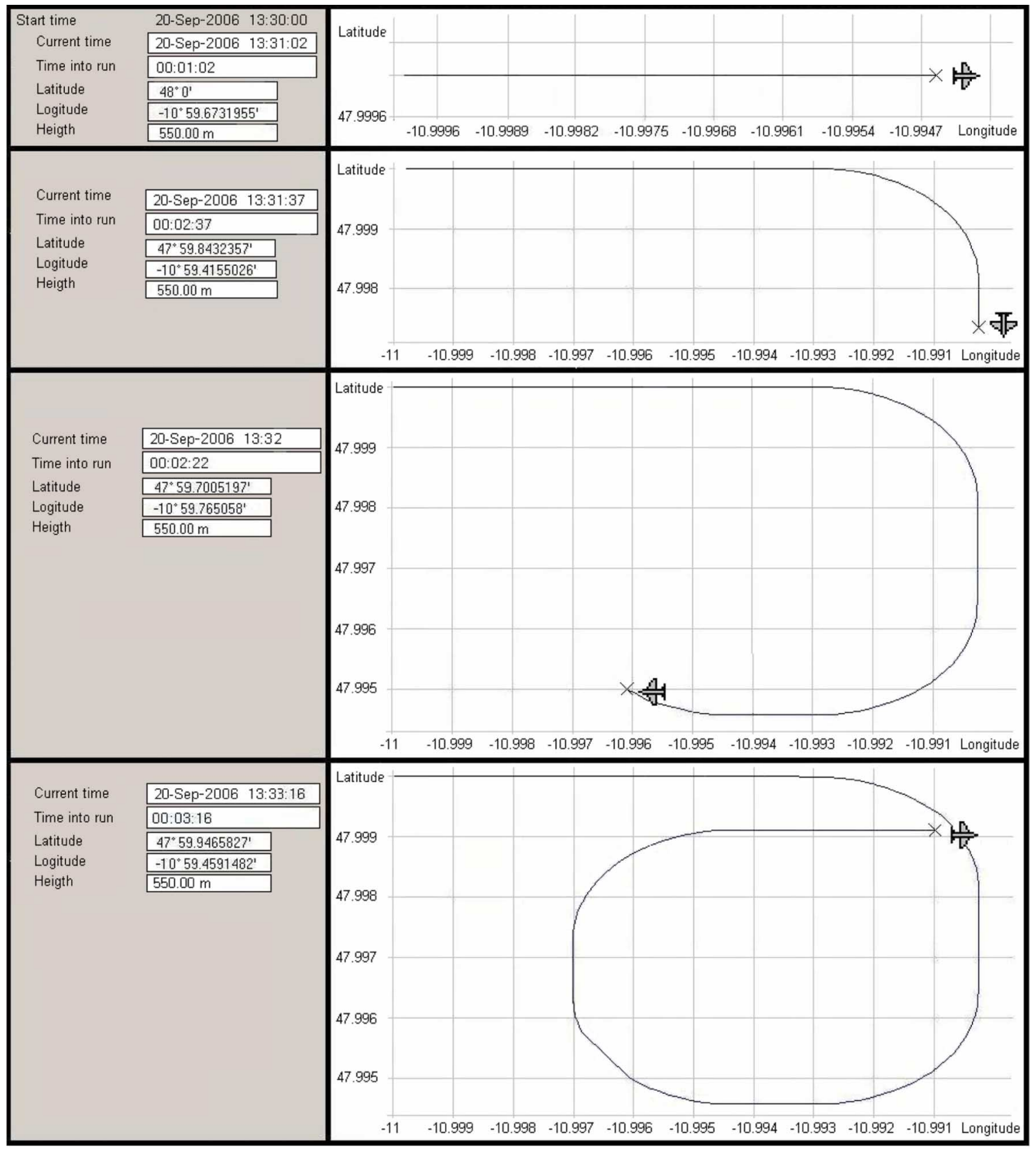

Fig. 10. Track defined by simulator at different times.

rising the power provided by the simulator. The measurement setup is shown in Fig. 4.

Figs. 10 and 11 show snapshots of simulated and measured heading, position, and user track for different simulation times. They demonstrate that the measurements follow exactly the simulated reference track including the correct heading information. The receiver had no preknowledge of the scenario and was able to derive all information from the RF signals provided by the simulator. It was also able to determine the position and spacing of the two antennas from the RF signals by its own autocalibration function after it had acquired the signals.

\section{B. DOA Estimation}

Direction finding is often used in adaptive antenna systems for controlling the positions of the array beam and/or spatial nulls. In the following, we present simulation results obtained with the use of MASTER for DOA estimation of a GPS L1 civil signal using estimation of signal parameters via rotation invariance technique (ESPRIT) [18]. For enabling the simulations, the ESPRIT algorithm has been implemented in a software-defined GPS receiver. The receiver operates on digital IF signals which are stored as binary data on the PC hard disc. Using the recorded binary signal data, the software receiver processes GPS signals on a PC in a way that is similar to the one in common GPS hardware. The MASTER environment with the wavefront MATRIX generator was used to simulate the outputs of a 2-by-2 uniform rectangular antenna array (URA) with half-wavelength element spacing. The simulated array outputs were recorded with the help of the NordNav "Quad Front End" as described in Section III-C (see also Fig. 8). Nominal power 


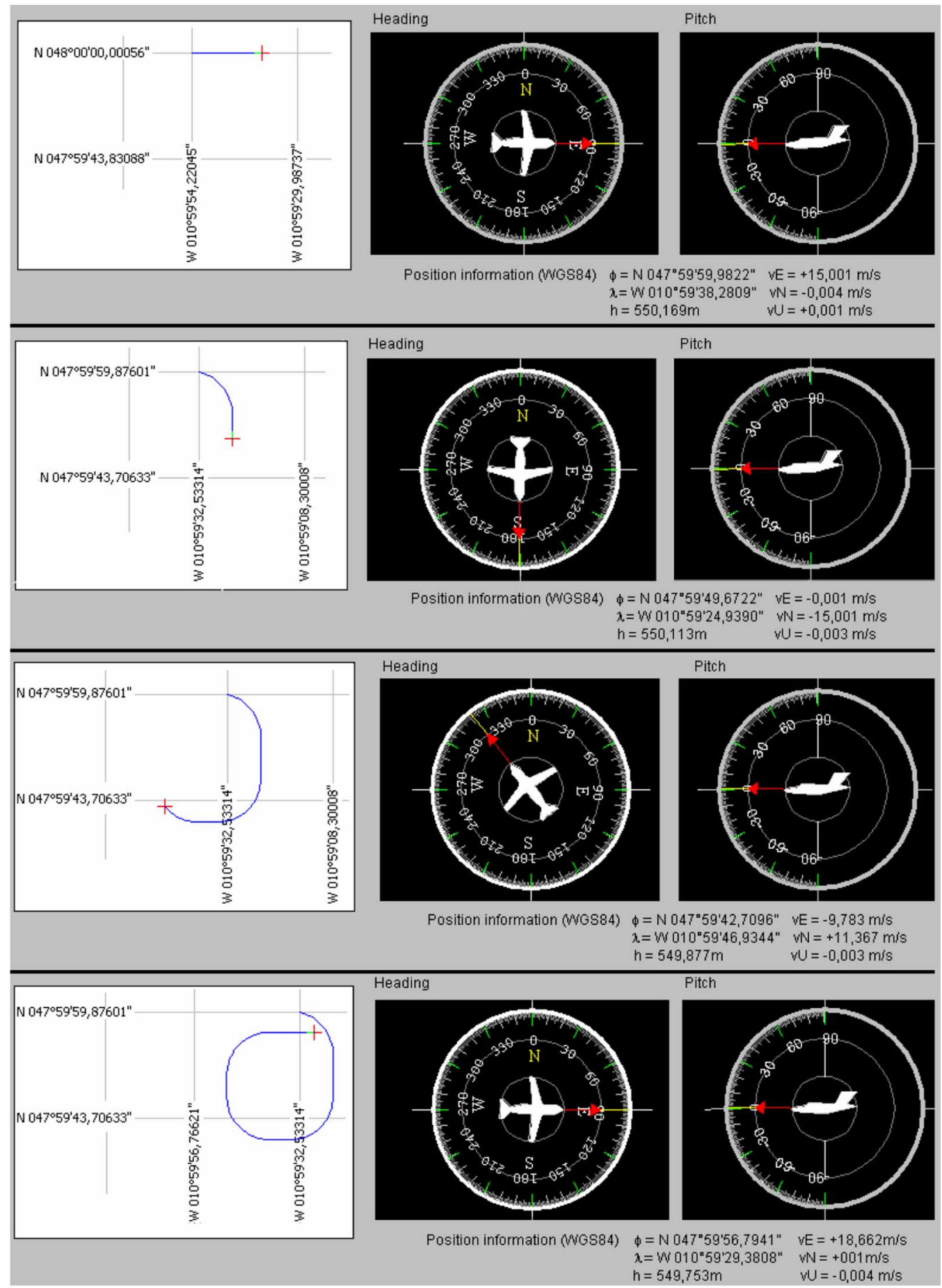

Fig. 11. Track and orientation calculated by the PolaRx2eH receiver from measured signals at same times as in Fig. 10. The heading information is illustrated by the receiver SW by the airplane symbol.

levels of GPS signals corresponding to the carrier-to-noise ratio of $45 \mathrm{~dB}-\mathrm{Hz}$ were adopted for ten simulated navigation satellite signals with PRNs from 1 to 10 . The ESPRIT algorithm was applied after the PRN-code correlation process (i.e., after signal despreading) and operated on sample blocks of the outputs of the I\&Q PRN-code correlators (see Fig. 6). There is one block of I\&Q correlators consisting of early, prompt, and late correlators after each of four antenna array elements. After transition time at the beginning of signal tracking, the PRN-code despreading gain is the highest at the prompt correlator channel and, there- fore, the outputs of the prompt correlators were used for direction finding. The integration time in the PRN-code correlators was set to $1 \mathrm{~ms}$ and a single observation of the correlator outputs formed a four-element vector of I\&Q samples. The adopted length of the data block used with the ESPRIT algorithm for estimating the DOA of each of ten simulated satellite signals was 70 observations, i.e., a data block size of 4-by-70 I\&Q samples, which corresponds to the algorithm observation time of $70 \mathrm{~ms}$.

As described in Section III-B, the carrier phase offsets between individual RF-channels are not calibrated internally by 


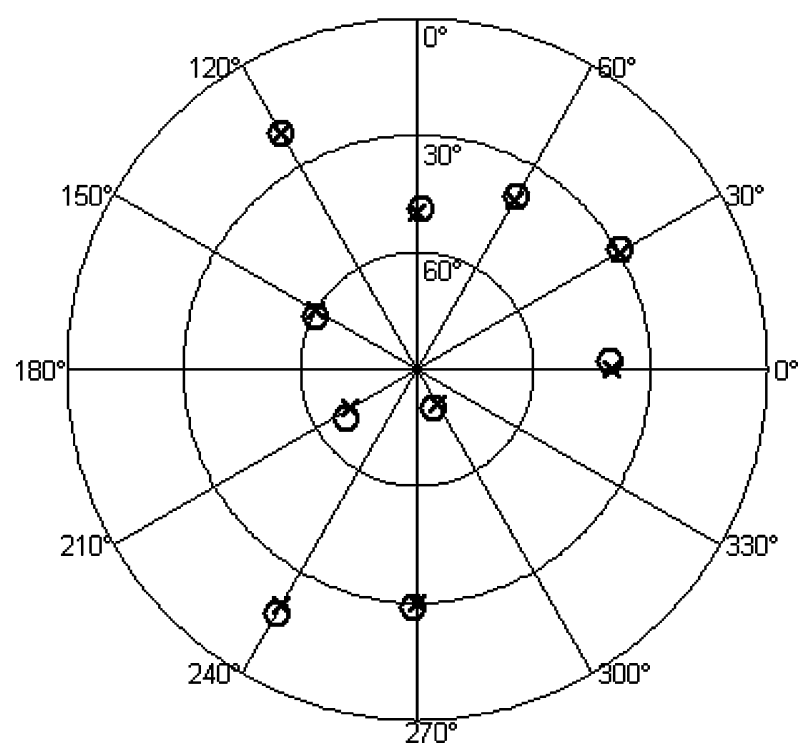

Fig. 12. Results of direction finding utilizing the ESPRIT algorithm, crosses denote the directions of arrival simulated by MASTER, circles denote the directions estimated with ESPRIT.

the MASTER hardware. This poses a problem for adaptive antenna applications as these hardware phase offsets distort the signal wavefronts simulated by MATRIX and lead to unpredictable results in direction finding and beamforming. The same effect is caused by the inter-channel phase biases in a multi-antenna RF front end. In order to avoid these harmful effects, the accumulated inter-channel phase biases of MASTER and the RF front end hardware should be calibrated and a set of correction coefficients applied in each antenna channel to compensate for the phase biases has to be generated. The approach with the use of the software GPS receiver described in Section III-B has been utilized for this purpose. Ten different sets of the carrier phase corrections were obtained by processing ten simulated GPS satellite signals. Each set obtained in this way provides, when applied, a perfect matching of direction finding for a signal with a specific PRN-code but may result in small mismatch for signals with other PRNs. For more general correction, a single set of phase corrections was produced by averaging over the obtained PRN-specific ten sets. This single set of phase corrections was then used with the DOA estimation of the simulated GPS signals. The results of direction finding are presented in Fig. 12, where good matching between simulated and detected DOAs for all ten GPS signals can be observed. Only small deviations between the reference and estimated directions occur when using the common set of carrier phase corrections.

\section{Interference Mitigation}

The testing of interference mitigation antenna algorithms is one of the prospective applications of MASTER. Hereafter, an illustrative example of testing an antenna beamforming algorithm for cancelling strong radio interference will be presented. The same experimental setup as in the case of direction finding (see Section IV-B) is used here, except that additionally to the GPS satellite signals one interferer signal has been generated.

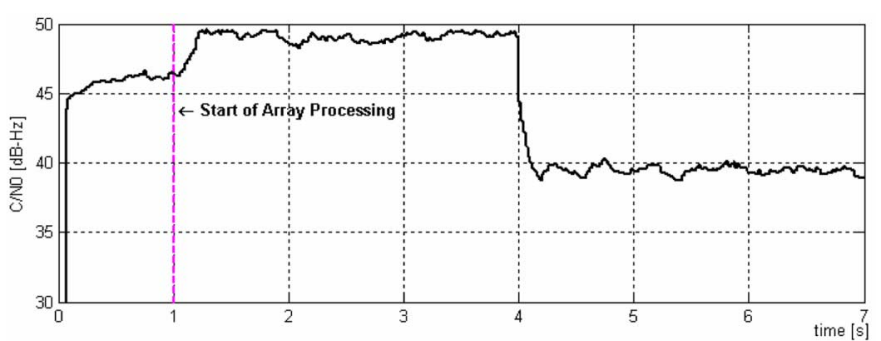

a)

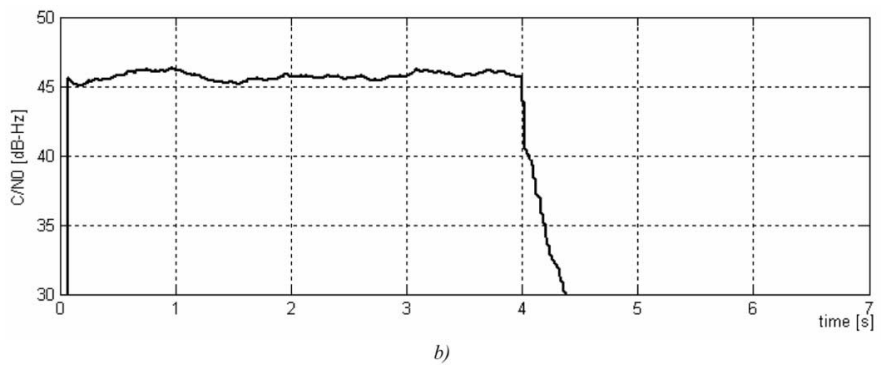

Fig. 13. Carrier-to-noise ratio of useful GPS signal (a) with and (b) without utilizing interference mitigation technique. In the case of (a), a 2-by-2 antenna array is used, in the case of (b), a single antenna.

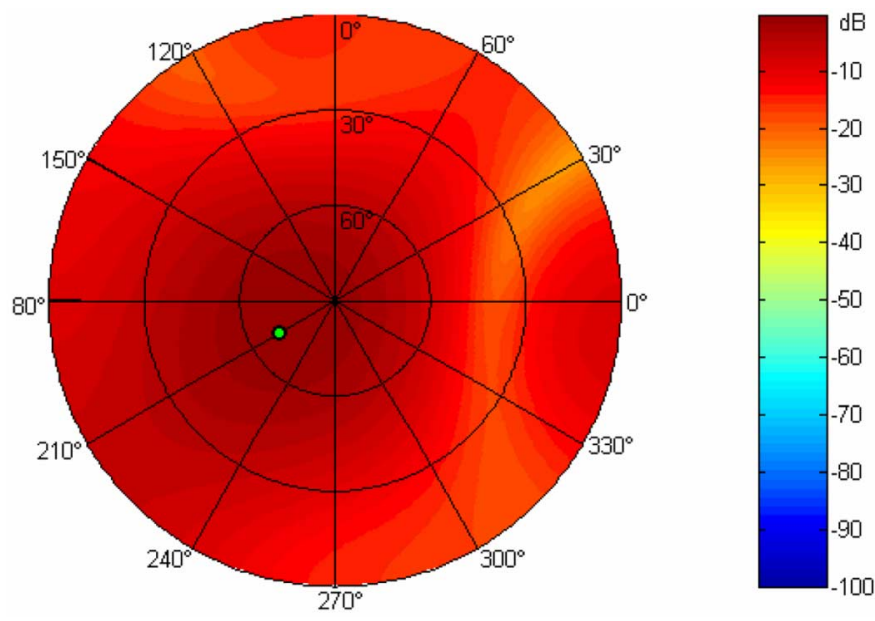

Fig. 14. Array gain pattern obtained with interference mitigation techniques for a 2-by-2 antenna array.

The interferer is a band-limited Gaussian noise with a bandwidth larger than the bandwidth of the RF frond end and a power level corresponding to $20 \mathrm{~dB}$ of interference-to-noise ratio.

The results for interference cancellation when tracking GPS signal with PRN 7 in the software receiver are presented in Figs. 13 and 14. The evolution of the carrier-to-noise ratio $\left(C / N_{0}\right)$ of the useful GPS signal can be seen in Fig. 13. The first $4 \mathrm{~s}$ at the beginning of the signal record used in this experiment do not contain radio interference and so the receiver was able to acquire the GPS signal and start to track it in a nominal signal condition. Please note that the $C / N_{0}$ values between the single antenna and antenna array receiver differ by approximately $4 \mathrm{~dB}$, which is due to the array gain produced by the beamforming using a linearly constrained minimum variance (LCMV) algorithm [3], [4]. This algorithm is used on the premise that the DOA of the useful GPS signal is known, which in practice can be fulfilled by using the GPS system almanac containing the information about the satellite orbits 
(satellite ephemeris). The beamformer weights are obtained in the way to direct the main beam towards the $k$ th GPS satellite, $1 \leq k \leq 10$, with a fixed gain and minimize the output power after beamforming

$$
\begin{aligned}
\min _{\mathbf{w}} & \mathbf{w}^{H} \mathbf{R}_{x x} \mathbf{w} \\
\text { subject to } & \mathbf{a}\left(\phi_{k, G P S}, \vartheta_{k, G P S}\right) \mathbf{w}=1
\end{aligned}
$$

where $\mathbf{w}$ is the vector of beamforming weights, $\mathbf{R}_{x x}$ is the array covariance matrix, $\mathbf{R}_{x x}=E\left\{\mathbf{x}[n] \mathbf{x}^{H}[n]\right\}$, and $E\{\cdot\}$ denotes the statistical expectation.

The LCMV algorithm has been implemented in a software defined GPS receiver after PRN-code correlation and uses a blockwise estimation of the array covariance matrix $\mathbf{R}_{x x}$ with the block length corresponding to $50-\mathrm{ms}$ observation time. The theoretically possible maximum array gain of $6 \mathrm{~dB}$ was not reached by the LCMV algorithm because of imperfections in the multi-antenna RF front end hardware.

When the interference signal is switched on, the single antenna receiver fails to keep tracking of the useful GPS signal but the antenna array receiver, although it experiences $10 \mathrm{~dB}$ drop of $C / N_{0}$, still is able to track the signal. The array gain pattern obtained with the help of the LCMV algorithm is shown in Fig. 14, the DOA of the GPS signal is marked with a circle. Please note that cancelling of the strong interference was produced by the LCMV technique without posing an additional spatial null constraint but through the adaptive minimization of the beamforming output power.

\section{Maximum Likelihood Parameter Estimation Using the SAGE Algorithm}

In order to achieve precise synchronization in a GNSS receiver with an antenna array even under presence of severe multi-path, we follow a maximum likelihood approach estimating $\boldsymbol{\theta}=\left[\boldsymbol{\theta}_{1}^{T}, \boldsymbol{\theta}_{2}^{T}, \ldots, \boldsymbol{\theta}_{L}^{T}\right]^{T}$ with $\boldsymbol{\theta}_{\ell}=\left[\gamma_{\ell}, \tau_{\ell}, \phi_{\ell}, \vartheta_{\ell}\right]^{T}$, according to the data model presented in Section II. Whereas no closed-form expression can be found for this maximum likelihood estimator (MLE), a numerical approach employs either a grid search or an iterative maximization of the likelihood function. In this paper, we explore the potential application of an iterative method for estimating the parameters of all impinging wavefronts in temporally and spatially white Gaussian noise: the space alternating generalized expectation-maximization (SAGE) algorithm. The SAGE algorithm [21] can be considered as a generalization of the well-known expectation-maximization (EM) algorithm [19], [20]. The potential of the SAGE algorithm in communication systems has been proven in [22], where it is shown that SAGE is a promising candidate for channel estimation in direct-sequence code division multiple access systems (DS-CDMA). The application of the SAGE algorithm for precise delay estimation in a GNSS receiver has been assessed in previous work [23]. The intention of this paper is to serve as a preliminary study on the performance of the algorithm under severe multi-path conditions using recorded data from a hardware signal generator as described in Section III-C.

The basic concept of the SAGE algorithm is the hidden data space [21]. Instead of estimating the parameters of all waves $\boldsymbol{\theta}$ in parallel within one iteration step as done by the EM algorithm,
TABLE I

CONSTELLATION

\begin{tabular}{|c|c|c|c|c|}
\hline SV & Azimuth & Elevation & $\Delta \tau$ & SMR \\
\hline 1 & $0^{\circ}$ & $40^{\circ}$ & - & - \\
\hline 1 (MP) & $180^{\circ}$ & $40^{\circ}$ & $0.1-1.1$ chip & $5 \mathrm{~dB}$ \\
\hline 2 & $30^{\circ}$ & $30^{\circ}$ & - & - \\
\hline 3 & $60^{\circ}$ & $40^{\circ}$ & - & - \\
\hline 4 & $90^{\circ}$ & $50^{\circ}$ & - & - \\
\hline 5 & $120^{\circ}$ & $20^{\circ}$ & - & - \\
\hline 6 & $150^{\circ}$ & $60^{\circ}$ & - & - \\
\hline 7 & $210^{\circ}$ & $70^{\circ}$ & - & - \\
\hline 8 & $240^{\circ}$ & $20^{\circ}$ & - & - \\
\hline 9 & $270^{\circ}$ & $30^{\circ}$ & - & - \\
\hline 10 & $300^{\circ}$ & $80^{\circ}$ & - & - \\
\hline
\end{tabular}

the SAGE algorithm estimates the parameters of each wave $\boldsymbol{\theta}_{\ell}$ sequentially. Also, instead of estimating the complete $\boldsymbol{\theta}_{\ell}$, SAGE breaks down the multi-dimensional optimization problem into several smaller problems by conditioning sequentially on a subspace of parameters, while keeping the parameters of the complement subspace fixed. We choose the hidden data space as one noisy wavefront. This choice of the hidden data space leads to a fast convergence rate and low complexity due to sequential updating and 1-D optimization procedures. Various choices of the hidden data space could be made which would individually influence the convergence rate [21]. Although the resulting log-likelihood values form a monotonically nondecreasing sequence, convergence to even a local maximum is not guaranteed. Therefore, the SAGE algorithm has to be initialized in a region which is close enough to a local (at best the global) maximum. Then, the sequence of estimates will converge in norm to it [21], [22]. Initialization is performed by sequential interference cancellation as described in [22] and [23].

We recorded data with the general setup as described in Section III-C. Additionally, a down conversion of the recorded data to baseband was performed applying a polyphase digital low pass filter and performing a sampling reduction. After filtering the one-sided bandwidth of the signal is $B=1.023 \mathrm{MHz}$ and the sampling frequency is $f_{s}=2 \cdot B$. In the following, we consider a two-path case $L=2$ and the parameters with the subscript $1, \boldsymbol{\theta}_{\mathbf{1}}=\left[\gamma_{1}, \tau_{1}, \phi_{1}, \vartheta_{1}\right]^{T}$ denote the parameters of the line-of-sight signal (LOSS). The parameters with the subscript $2, \boldsymbol{\theta}_{\mathbf{2}}=\left[\gamma_{2}, \tau_{2}, \phi_{2}, \vartheta_{2}\right]^{T}$ stand for the reflection. We simulated a URA with $M=M_{x} \cdot M_{y}=2 \cdot 2=4$. The relative time-delay between the LOSS and the multi-path in chips is referred to as $\Delta \tau=\left|\tau_{1}-\tau_{2}\right| / T_{c}$. We assume a static channel with the multi-path signal being in-phase with the LOSS, SMR $=5$ $\mathrm{dB}$ (signal-to-multi-path ratio), and $C / N_{0}=42 \mathrm{~dB}-\mathrm{Hz}$ for the LOSS. We simulated the constellation of satellite vehicles (SV) and one multi-path signal of SV 1 denoted 1 (MP) as shown in Table I.

Unfortunately, we do not obtain an absolute reference for the delay estimates, because the recording of the data through the NordNav "Quad Front End" had to be started manually. Thus, we additionally simulated a reference scenario with no multi-path present at the beginning of each scenario as defined in Table I. We recorded one scenario of approximately $80 \mathrm{~s}$ length for each relative delay $\Delta \tau(0.1-1.1$ chip $)$ with the first $20 \mathrm{~s}$ without multi-path and then the multi-path was "switched on." Both, the reference scenario without multi-path (the first 


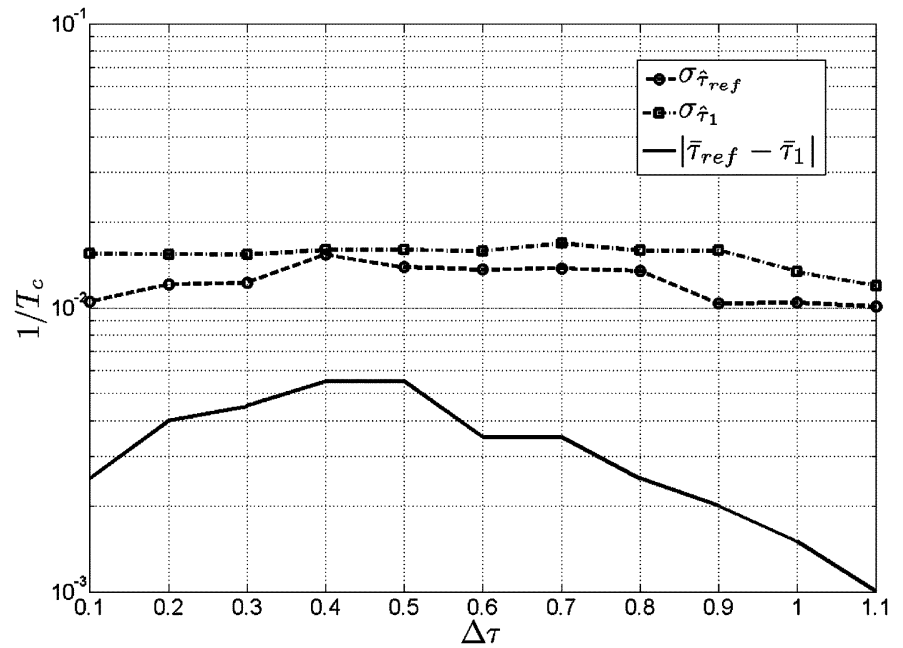

Fig. 15. Delay estimation of the LOSS versus the relative time-delay $\Delta \tau$. Parameters: $L=2, M=9, \phi_{1}=0^{\circ}, \vartheta_{1}=40^{\circ}, \phi_{2}=180^{\circ}, \vartheta_{2}=40^{\circ}$.

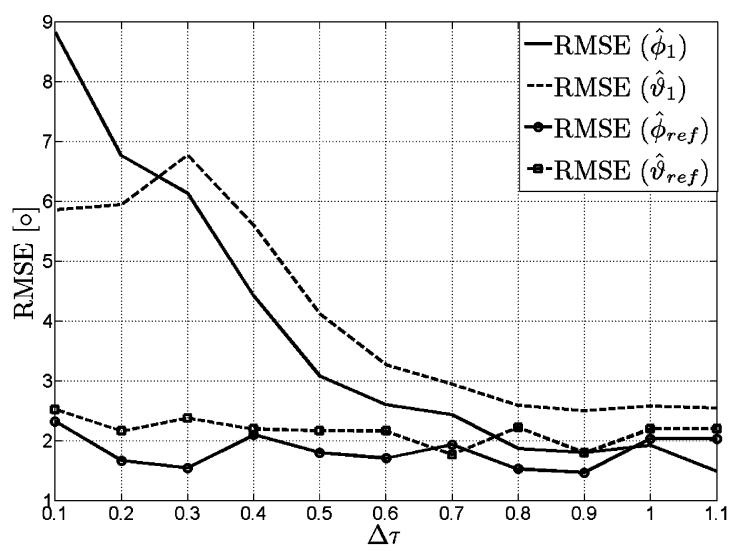

Fig. 16. RMSE of the azimuth and elevation in degrees of the LOSS versus the relative time-delay $\Delta \tau$. Parameters: $L=2, M=9, \phi_{1}=0^{\circ}, \vartheta_{2}=40^{\circ}$.

$20 \mathrm{~s}$ of each recording) and the scenario with multi-path (the following $60 \mathrm{~s}$ ) are processed by the SAGE algorithm in order to obtain parameter estimates for the signal parameters for the $\operatorname{LOSS} \hat{\boldsymbol{\theta}}_{1}=\left[\hat{\gamma}_{1}, \hat{\tau}_{1}, \hat{\phi}_{1}, \hat{\vartheta}_{1}\right]^{T}$ and for the reflective multi-path $\hat{\boldsymbol{\theta}}_{2}=\left[\hat{\gamma}_{2}, \hat{\tau}_{2}, \hat{\phi}_{2}, \hat{\vartheta}_{2}\right]^{T}$. The delay estimates of the reference scenarios shall serve as reference for the delay estimates of the scenarios with multi-path. The observation time we choose to $20 \mathrm{~ms}$. In other words, this means that for an estimate of $\boldsymbol{\theta}, 20 \mathrm{~ms}$ of data are used. Fig. 15 depicts the standard deviation of the delay estimates of the LOSS for the reference scenarios $\sigma_{\hat{\tau}_{\text {ref }}}$, the standard deviation of the delay estimates of the LOSS for the multi-path scenarios $\sigma_{\hat{\tau}_{1}}$, and the bias of the mean value of the estimation of the delay of the LOSS for the reference scenarios and the multi-path scenarios $\left|\bar{\tau}_{\text {ref }}-\bar{\tau}_{1}\right|$. Fig. 16 illustrates the root mean square estimation (RMSE) error of the azimuth and the elevation of the reference scenarios and the multi-path scenarios. Fig. 17 depicts the mean number of iteration cycles $\bar{k}$. In general, the behavior of the SAGE algorithm for the recorded multi-path scenario is rather similar to the behavior with simulated signals as in [23] and [25]. The RMSE for the azimuth and the elevation for small $\Delta \tau$ as given in Fig. 16 become biased

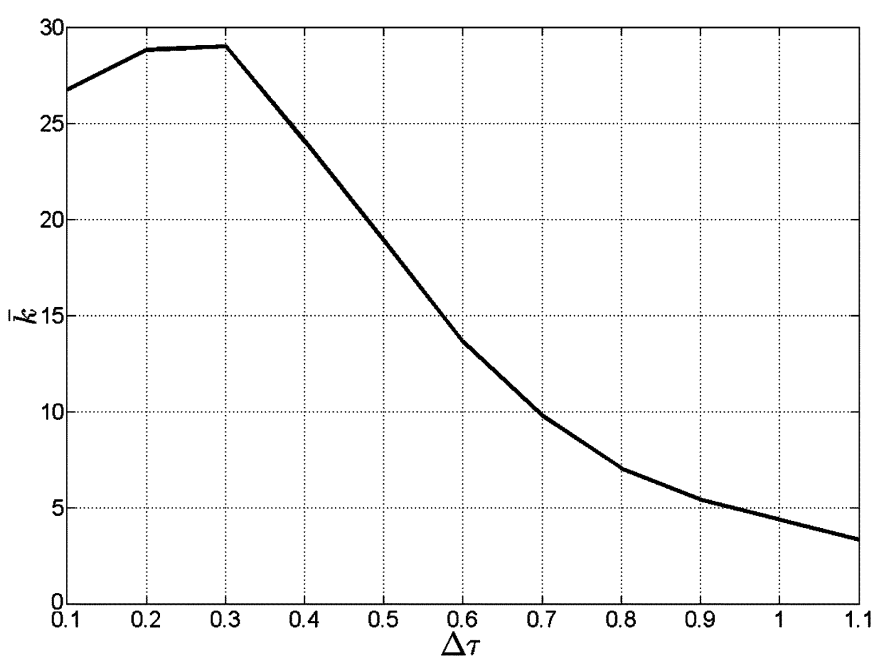

Fig. 17. Mean number of iteration cycles versus the relative time-delay $\Delta \tau$. Parameters: $L=2, M=9, \phi_{1}=0^{\circ}, \phi_{2}=180^{\circ}, \vartheta_{2}=40^{\circ}$.

due to the limited resolution of quantization of only $2 \mathrm{~b}$ and calibration errors.

\section{CONCLUSION}

The concept of MASTER has been presented. The realization of the approach for wavefront generation at digital baseband level for multiple GNSS signals arriving from different directions has been described in details. It has been shown that the use of the MASTER concept offers the possibility to test receivers with multiple antenna inputs and array processing algorithms in a small laboratory in realistic and repeatable GNSS signal environments without the need for measurements in an anechoic chamber or building complicated RF signal combining networks. The current version of MASTER utilizes the narrowband signal assumption and, therefore, the direction of arrivals are simulated by adapting the carrier phases of a signal to different equivalent (virtual) antenna RF outputs.

The use of the MASTER environment for algorithm testing in prospective applications has been demonstrated by examples of different applications: DOA finding, beamforming for radio interference mitigation, and estimation of GNSS signal parameters in an antenna-array receiver with SAGE, as well as a heading algorithm in a dual-antenna receiver. The antenna array algorithms show good test results. The phase calibration of MASTER can be performed with sufficient accuracy of approximately $3^{\circ}$. However, it became obvious that DOA estimation and beamforming with spatial reference are very sensitive to the calibration of the multiple antenna front end with respect to the signal carrier phase, even if the RF outputs of the simulator are carefully calibrated. A method for phase calibration of the antenna front end by data post processing has been also presented in this paper.

Some of the tested algorithms are less sensitive to phase calibration as, for example, the SAGE algorithm for signal parameter estimation or the heading algorithm in the PolaRx2eH dualantenna GPS receiver, which applies double differencing techniques to the measured carrier phases. Similarly, some adaptive array algorithms can be applied to improve the signal reception 
without knowledge of the DOAs for useful and interference signals. In summary, the results of the performed tests show that MASTER is a very powerful tool for testing of multi-antenna or antenna array applications in satellite navigation.

\section{REFERENCES}

[1] A. Hornbostel, H. Denks, and H. Venus, "First results of baseband wavefront generation with a digital channel matrix for testing of CRPA," presented at the ION GNSS, Fort Worth, TX, Sep. 2006.

[2] S. Bellofiore et al., "Smart-antenna systems for mobile communications network part 2: Beamforming and network throughput," IEEE Ant. Propag. Mag., vol. 44, no. 4, pp. 106-114, Aug. 2002.

[3] H. L. Van Trees, Optimum Array Processing. Hoboken, NJ: Wiley, 2002.

[4] K. Litva and Y. Lo, Digital Beamforming in Wireless Communications. Norwood, MA: Artech House, 1996.

[5] A. Konovaltsev, B. Belabbas, H. Denks, and A. Hornbostel, "Adaptive antenna against multipath?," presented at the ENC-GNSS, Rotterdam, Netherlands, May 2004.

[6] G. G. Seco, "Antenna Arrays for Multipath and Interference Mitigation in GNSS Receivers," Ph.D. dissertation, Dept. Signal Theory Commun., Univ. Politecnica Catalunya, Barcelona, Spain, 2000.

[7] G. S. Granados, J. A. Fernandez-Rubio, and C. F. Prades, "ML estimator and hybrid beamformer for multipath and interference mitigation in GNSS receivers," IEEE Trans. Signal Process., vol. 53, no. 3, pp. 1194-1208, Mar. 2005.

[8] J. S. Vera, "Subspace methods to multipath mitigation in a navigation receiver," in Proc IEEE Veh. Techn. Conf. (VTC), 1999, pp. 2077-2081.

[9] J. S. Vera, "Efficient multipath mitigation in navigation systems," Ph.D. dissertation, Dept. Signal Theory Commun., Univ. Politecnica Catalunya, Barcelona, Spain, 2004.

[10] J. S. Vera, "An efficient Newton-type method for the computation of ML estimators in a uniform linear array," IEEE Trans. Signal Process., vol. 53, no. 6, pp. 2036-2045, Jun. 2005.

[11] C. F. Prades, "Advanced signal processing techniques for global navigation satellite systems receivers," Ph.D. dissertation, Dept. Signal Theory Commun., Univ. Politecnica Catalunya, Barcelona, Spain, 2005.

[12] C. F. Prades, J. A. F. Rubio, and G. S. Granados, "On the equivalence of joint maximum likelihood approach and the multiple hybrid beamforming in GNSS synchronization," in Proc. 6th Baiona Workshop Signal Process. Commun., 2003, pp. 173-177.

[13] D. de Lorenzo, J. Rife, P. Enge, and D. Akos, "Navigation accuracy and interference rejection for an adaptive GPS antenna array," presented at the ION GNSS, Fort Worth, TX, Sep. 2006.

[14] D. d. Lorenzo, J. Gautier, J. Rife, P. Enge, and D. Akos, "Adaptive array processing for GPS interference rejection," presented at the ION GNSS, Long Beach, CA, Sep. 2006.

[15] L. V. Kuylen, F. Boon, and A. Simsky, "Attitude determination methods used in the Polarx2@ multi-antenna GPS receiver," presented at the ION GNSS, Long Beach, CA, Sep. 2005.

[16] A. Simsky, L. V. Kuylen, and F. Boon, "Single-board attitude determination system based on the PolaRx2@ GPS receiver," presented at the ENC GNSS, Munich, Germany, Jul. 2005.

[17] Septentrio, Leuven, Belgium, "PolaRx2 Version 2.6, user manual," 2005.

[18] M. Haardt, M. D. Zoltowski, and C. P. Mathews, "ESPRIT and closedform 2D angle estimation with planar arrays," in Digital Signal Processing Handbook, V. Madisetti and D. Williams, Eds. Boca Raton, FL: CRC Press, 1998.

[19] A. P. Dempster, N. M. Laird, and D. B. Rubin, "Maximum likelihood from incomplete data via the EM algorithm," J. Royal Statistical Soc. B., vol. 39, no. 1, pp. 1-38, 1977.

[20] T. K. Moon, "The expectation maximization algorithm," IEEE Signal Process. Mag., vol. 13, no. 6, pp. 47-60, Nov. 1996.

[21] J. A. Fessler and A. O. Hero, "Space-alternating generalized expectation-maximization algorithm," IEEE Trans. Signal Process., vol. 42, no. 10, pp. 2664-2677, Oct. 1994.
[22] B. H. Fleury, M. Tschudin, R. Heddergott, D. Dahlhaus, and K. I. Pedersen, "Channel parameter estimation in mobile radio environments using the SAGE algorithm," IEEE JSAC Wirel. Commun. Series, vol. 17, no. 3, pp. 434-450, Mar. 1999.

[23] F. Antreich, O. E. Rodriguez, J. A. Nossek, and W. Utschick, "Estimation of synchronization parameters using SAGE in a GNSS-receiver," presented at the ION GNSS, Long Beach, CA, Sep. 2005.

[24] B. W. Parkinson and J. J. Spilker, Eds., "Global Positioning System: Theory and Applications," in Progress in Astronautics and Aeronautics. New York: AIAA, 1996, vol. 1.

[25] F. Antreich, J. A. Nossek, and W. Utschick, "Maximum likelihood delay estimation in a navigation receiver for aeronautical applications," Aerosp. Sci. Technol., accepted for publication.

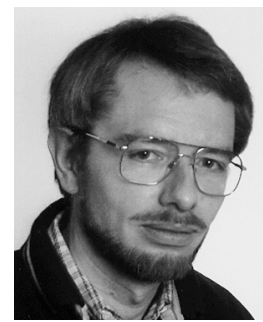

Achim Hornbostel received the diploma in electrical engineering and the $\mathrm{Ph} . \mathrm{D}$. degree in electrical engineering from the University of Hannover, Hannover, Germany, in 1989 and 1995, respectively.

In 2000 , he became a member of the staff of the Institute of Communications and Navigation, DLR, Wessling-Oberpfaffenhofen, Germany, where he is currently leading a working group for receivers and algorithms since 2005. In 1989, he joined the German Aerospace Center (DLR). He was involved in several projects for remote sensing, satellite communications, and satellite navigation. His main research activities are currently in signal propagation and receiver development.

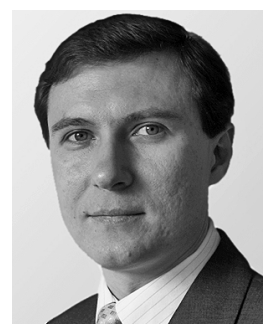

Andriy Konovaltsev received the diploma and $\mathrm{Ph} . \mathrm{D}$. degrees in radio engineering from the State Technical University of Radio Electronics (KhTURE), Kharkov, Ukraine, in 1993 and 1996, respectively.

From 1997 to 2000, he was Research Assistant with KhTURE. He joined the Institute of Communications and Navigation of the German Aerospace Center (DLR), Wessling-Oberpfaffenhofen, Germany, in 2001. His current research interests include array processing for satellite navigation systems, signal processing algorithms for navigation receivers including synchronization, multipath, and radio interference mitigation.

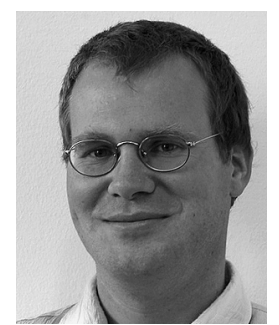

Holmer Denks received the diploma in electrical engineering and communications from the University of Kiel, Kiel, Germany, in 2002.

In 2002, he joined the Institute of Communications and Navigation of the German Aerospace Center (DLR), Wessling-Oberpfaffenhofen, Germany, where he currently works on simulations of Galileo signals. He was involved in investigation and simulation of communications systems.

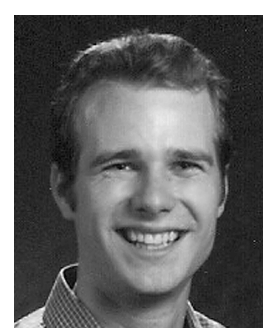

Felix Antreich (M'06) received the diploma in electrical engineering from the Munich University of Technology (TUM), Munich, Germany, in 2003, where he is currently pursuing the Ph.D. degree in electrical engineering.

Since July 2003, he has been with the Institute of Communications and Navigation of the German Aerospace Center (DLR), Wessling-Oberpfaffenhofen, Germany. 